\title{
A Birkhoff connection between quantum circuits and linear classical reversible circuits
}

\author{
Alexis De Vos ${ }^{1}$ and Stijn De Baerdemacker ${ }^{2}$ \\ ${ }^{1}$ Universiteit Gent, B - 9000 Gent, Belgium \\ vakgroep elektronica en informatiesystemen \\ alexis.devos@ugent.be \\ ${ }^{2}$ University of New Brunswick, Fredericton E3B 5A3, Canada \\ department of chemistry
}

\begin{abstract}
Birkhoff's theorem tells how any doubly stochastic matrix can be decomposed as a weighted sum of permutation matrices. Similar theorems on unitary matrices reveal a connection between quantum circuits and linear classical reversible circuits. It triggers the question whether a quantum computer can be regarded as a superposition of classical reversible computers.
\end{abstract}

\section{Introduction}

Let $D$ be an arbitrary $n \times n$ doubly stochastic matrix. This means that all entries $D_{j k}$ are real and satisfy $0 \leq D_{j k} \leq 1$ and that all line sums (i.e. the $n$ row sums and the $n$ column sums) are equal to 1 . Let $\mathrm{P}(n)$ be the group of $n \times n$ permutation matrices. Birkhoff [1] has demonstrated

Theorem 1 Any $n \times n$ doubly stochastic matrix $D$ can be written

$$
D=\sum_{j} c_{j} P_{j}
$$

with all $P_{j} \in P(n)$ and the weights $c_{j}$ real, satisfying both $0 \leq c_{j} \leq 1$ and $\sum_{j} c_{j}=1$.

Because unitary matrices describe quantum circuits [2] and permutation matrices describe classical reversible circuits [3], the question arises whether a similar theorem holds for matrices from the unitary group $\mathrm{U}(n)$. In a sloppy way, one might reformulate the question as:

Is a quantum computer a quantum superposition of a finite number of classical (reversible) computers?

It is a surprise that (to our knowledge) this problem has not been discussed in the literature. 
It is clear that a simple positive answer to the above question is not possible. Indeed, any sum $\sum_{j} c_{j} P_{j}$ is a matrix with identical line sums (equal to $\sum_{j} c_{j}$ ), whereas an arbitrary unitary matrix usually does not have identical line sums. Moreover, if all $c_{j}$ are real, then the matrix $\sum_{j} c_{j} P_{j}$ has exclusively real entries, again a property not shown by an arbitrary unitary matrix. Nevertheless, below we will present some Birkhoff-like theorems concerning $n \times n$ unitary matrices in general and $2^{w} \times 2^{w}$ unitary matrices in peculiar.

\section{The ZXZ decomposition of a unitary matrix}

Each quantum circuit acting on $w$ qubits is represented by a $2^{w} \times 2^{w}$ unitary matrix. Such matrix thus is a member of the unitary group $\mathrm{U}(n)$ with $n=2^{w}$. In light of quantum circuit decomposition, the (sub)group structure of $\mathrm{U}(n)$ is particularly important. We note the following two useful subgroups [4] [5]:

- $\mathrm{XU}(n)$, i.e. the group of $\mathrm{U}(n)$ matrices with all line sums equal to 1 and

- $\mathrm{ZU}(n)$, i.e. the group of diagonal $\mathrm{U}(n)$ matrices with upper-left entry equal to 1.

Whereas $\mathrm{U}(n)$ is a group of dimension $n^{2}, \mathrm{XU}(n)$ is a group of dimension $(n-1)^{2}$ and $\mathrm{ZU}(n)$ is a group of dimension $n-1$.

Idel and Wolf [6] proved the following theorem:

Theorem 2 Every $n \times n$ unitary matrix $U$ can be decomposed as

$$
U=a Z_{1} X Z_{2}
$$

where both $Z_{1}$ and $Z_{2}$ are $Z U(n)$ matrices, where $X$ is an $X U(n)$ matrix, and $a$ is a unit-modulus scalar.

Proof of the theorem is based on simplectic topology and, unfortunately, is not constructive. There exists an iterative method [7] for, given a matrix $U$, finding a set $\left(a, Z_{1}, X, Z_{2}\right)$ with arbitrary numerical precision. If $n$ equals $2^{w}$, then the matrix decomposition expresses the decomposition of a quantum circuit acting on $w$ qubits [8]. The 3 -qubit case $(n=8)$ looks like

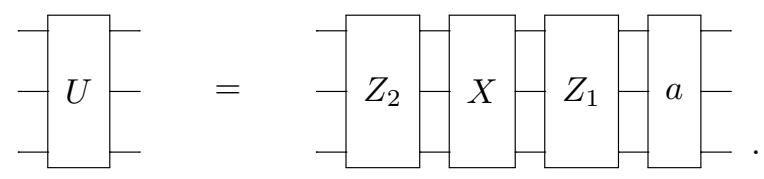

\section{The Birkhoff decomposition of the XU matrix}

De Baerdemacker et al. [9] proved the following theorem: 
Theorem 3 Every $X U(n)$ matrix $X$ can be decomposed as

$$
X=\sum_{j=1}^{n !} c_{j} P_{j},
$$

where $P_{j}$ are the $n \times n$ permutation matrices and $c_{j}$ are complex numbers, such that both $\sum c_{j}=1$ and $\sum\left|c_{j}\right|^{2}=1$.

De Baerdemacker et al. provide an algorithm to find any possible set of appropriate weights $c_{j}$. This set is far from unique (except if $n=2$ ).

De Vos and De Baerdemacker [10] [11] demonstrated, in case $n$ equals a power of 2 (say, $n=2^{w}$ ), the following theorem:

Theorem 4 Every $X U\left(2^{w}\right)$ matrix $X$ can be decomposed as

$$
X=\sum_{j=1}^{N(w)} c_{j} E_{j},
$$

where $j$ runs over all $2^{w} \times 2^{w}$ epicirculant permutation matrices $E_{j}$, where $c_{j}$ are complex numbers, such that both $\sum c_{j}=1$ and $\sum\left|c_{j}\right|^{2}=1$, and $N(w)$ equals $2^{w}\left(2^{w}-1\right)\left(2^{w}-2\right)\left(2^{w}-2^{2}\right) \ldots\left(2^{w}-2^{w-1}\right)$.

In next section will be explained what is meant with 'epicirculant matrix'. Theorem 4 is stronger than Theorem 3 , because $N(w)$ scales much better than $\left(2^{w}\right)$ ! for large $w$, as can be seen in the table:

\begin{tabular}{|r|rrr|}
\hline$w$ & $2^{w}$ & $\left(2^{w}\right) !$ & $N(w)$ \\
\hline 1 & 2 & 2 & 2 \\
2 & 4 & 24 & 24 \\
3 & 8 & 40,320 & 1,344 \\
4 & 16 & $20,922,789,888,000$ & 322,560 \\
\hline
\end{tabular}

One possible set of weights $c_{j}$ is given by

$$
c_{j}=\delta_{1, j}+\frac{2^{w}-1}{N(w)}\left[\operatorname{Trace}\left(E_{j}^{-1} X\right)-\operatorname{Trace}\left(E_{j}\right)\right],
$$

where the Kronecker delta assumes that the epicirculant matrix $E_{1}$ is the $2^{w} \times 2^{w}$ unit matrix.

\section{Epicirculant matrices}

Before giving the definition of a $2^{w} \times 2^{w}$ epicirculant matrix, it is useful to introduce some convenient conventions: 
Remark 1 In the present paper, rows and columns of any $2^{w} \times 2^{w}$ matrix are numbered from 0 to $2^{w}-1$ (instead of the conventional numbering from 1 to $2^{w}$ ) and each such number is represented by the $w \times 1$ matrix consisting of the $w$ bits of the binary notation of the row-or-column number.

E.g. the upper-left entry of the $8 \times 8$ matrix $A$ is entry $A_{0,0}=A_{(0,0,0)^{T},(0,0,0)^{T}}$, whereas its lower-right entry is denoted $A_{7,7}=A_{(1,1,1)^{T},(1,1,1)^{T}}$. Further, we choose to order bits from least significant to most significant bit. E.g., for $w=3$, the vector $(1,1,0)^{T}$ denotes the number 3 .

Definition 1 A $2^{w} \times 2^{w}$ epicirculant matrix $M$ is a $2^{w} \times 2^{w}$ matrix, such that each entry $M_{j, k}$ equals the entry $M_{0, c}$ with $c=k-x j$, where the multiplication $x j$ is a matrix multiplication performed modulo 2 , and where $x$ is some invertible $w \times w$ matrix with entries from $\{0,1\}$, called the pitch matrix. The subtraction $k-x j$ is a vector addition performed modulo 2.

E.g. the following matrix is an $8 \times 8$ epicirculant matrix with pitch matrix $x=\left(\begin{array}{lll}1 & 0 & 0 \\ 0 & 0 & 1 \\ 0 & 1 & 1\end{array}\right):$

$$
\left(\begin{array}{llllllll}
m_{0} & m_{1} & m_{2} & m_{3} & m_{4} & m_{5} & m_{6} & m_{7} \\
m_{1} & m_{0} & m_{3} & m_{2} & m_{5} & m_{4} & m_{7} & m_{6} \\
m_{4} & m_{5} & m_{6} & m_{7} & m_{0} & m_{1} & m_{2} & m_{3} \\
m_{5} & m_{4} & m_{7} & m_{6} & m_{1} & m_{0} & m_{3} & m_{2} \\
m_{6} & m_{7} & m_{4} & m_{5} & m_{2} & m_{3} & m_{0} & m_{1} \\
m_{7} & m_{6} & m_{5} & m_{4} & m_{3} & m_{2} & m_{1} & m_{0} \\
m_{2} & m_{3} & m_{0} & m_{1} & m_{6} & m_{7} & m_{4} & m_{5} \\
m_{3} & m_{2} & m_{1} & m_{0} & m_{7} & m_{6} & m_{5} & m_{4}
\end{array}\right) .
$$

Thanks to the fact that matrix $x$ is invertible, not only each of the eight rows but also each of the eight columns contains exactly one $m_{0}$, one $m_{1}, \ldots$, and one $m_{7}$. If all entries of its upper row (i.e. row 0 ) are equal to 0 , except one entry equal to 1 (in column $s$ ), then an epicirculant matrix is an epicirculant permutation matrix. The vector representing position $s$ is called the shift vector. There exist as many different epicirculant permutation matrices as there exist possible shift vectors (i.e. $2^{w}$ ) times the number of possible pitch matrices (i.e. $\left.\left(2^{w}-1\right)\left(2^{w}-2\right)\left(2^{w}-2^{2}\right) \ldots\left(2^{w}-2^{w-1}\right)\right)$. Because

- the shift vectors form a group isomorphic to the direct product $\left(\mathbf{C}_{2}\right)^{w}$ of $w$ cyclic groups, each of order 2 , and therefore of order $2^{w}$ and

- the pitch matrices form a group isomorphic to the general linear group $\operatorname{GL}(w, 2)$ of order $\left(2^{w}-1\right)\left(2^{w}-2\right)\left(2^{w}-2^{2}\right) \ldots\left(2^{w}-2^{w-1}\right)$,

the epicirculant permutation matrices form a group [12] isomorphic to the general affine group $\operatorname{GA}(w, 2)$ of order $N(w)$, isomorphic to the semidirect product $\left(\mathbf{C}_{2}\right)^{w}: \mathrm{GL}(w, 2)$. E.g. the following matrix is an $8 \times 8$ epicirculant permutation 
matrix with shift vector $s=\left(\begin{array}{l}0 \\ 1 \\ 0\end{array}\right)$ and pitch matrix $x=\left(\begin{array}{lll}1 & 0 & 0 \\ 0 & 0 & 1 \\ 0 & 1 & 1\end{array}\right)$ :

$$
\left(\begin{array}{llllllll}
0 & 0 & 1 & 0 & 0 & 0 & 0 & 0 \\
0 & 0 & 0 & 1 & 0 & 0 & 0 & 0 \\
0 & 0 & 0 & 0 & 0 & 0 & 1 & 0 \\
0 & 0 & 0 & 0 & 0 & 0 & 0 & 1 \\
0 & 0 & 0 & 0 & 1 & 0 & 0 & 0 \\
0 & 0 & 0 & 0 & 0 & 1 & 0 & 0 \\
1 & 0 & 0 & 0 & 0 & 0 & 0 & 0 \\
0 & 1 & 0 & 0 & 0 & 0 & 0 & 0
\end{array}\right) .
$$

It is obtained from matrix (2) by choosing $m_{2}=1$ and $m_{k}=0$ for $k \neq 2$. We note that, if $s$ is the $w \times 1$ zero matrix and $x$ is the $w \times w$ unit matrix, then the corresponding epicirculant permutation matrix is the $2^{w} \times 2^{w}$ unit matrix $E_{1}$.

We have [11]:

Lemma 1 Each epicirculant permutation matrix $E$ can be written as the product of a zero-shift epicirculant permutation matrix $L$ and a unit-pitch epicirculant permutation matrix $N$ :

$$
E=L N
$$

E.g. matrix (3) has the decomposition

$$
\left(\begin{array}{llllllll}
1 & 0 & 0 & 0 & 0 & 0 & 0 & 0 \\
0 & 1 & 0 & 0 & 0 & 0 & 0 & 0 \\
0 & 0 & 0 & 0 & 1 & 0 & 0 & 0 \\
0 & 0 & 0 & 0 & 0 & 1 & 0 & 0 \\
0 & 0 & 0 & 0 & 0 & 0 & 1 & 0 \\
0 & 0 & 0 & 0 & 0 & 0 & 0 & 1 \\
0 & 0 & 1 & 0 & 0 & 0 & 0 & 0 \\
0 & 0 & 0 & 1 & 0 & 0 & 0 & 0
\end{array}\right)\left(\begin{array}{llllllll}
0 & 0 & 1 & 0 & 0 & 0 & 0 & 0 \\
0 & 0 & 0 & 1 & 0 & 0 & 0 & 0 \\
1 & 0 & 0 & 0 & 0 & 0 & 0 & 0 \\
0 & 1 & 0 & 0 & 0 & 0 & 0 & 0 \\
0 & 0 & 0 & 0 & 0 & 0 & 1 & 0 \\
0 & 0 & 0 & 0 & 0 & 0 & 0 & 1 \\
0 & 0 & 0 & 0 & 1 & 0 & 0 & 0 \\
0 & 0 & 0 & 0 & 0 & 1 & 0 & 0
\end{array}\right) .
$$

The left matrix has shift equal to $\left(\begin{array}{l}0 \\ 0 \\ 0\end{array}\right)$ and pitch equal to $\left(\begin{array}{lll}1 & 0 & 0 \\ 0 & 0 & 1 \\ 0 & 1 & 1\end{array}\right)$, whereas the right matrix has shift vector $\left(\begin{array}{l}0 \\ 1 \\ 0\end{array}\right)$ and pitch matrix $\left(\begin{array}{lll}1 & 0 & 0 \\ 0 & 1 & 0 \\ 0 & 0 & 1\end{array}\right)$.

From classical reversible computation [3] [13] [14] [15], we know the following two lemmas:

Lemma 2 An arbitrary zero-shift epicirculant permutation matrix L represents a linear circuit, i.e. a circuit consisting exclusively of singly controlled NOT gates (a.k.a. FEYNMAN gates).

and

Lemma 3 An arbitrary unit-pitch epicirculant permutation matrix $N$ represents a circuit consisting merely of a stack of $w$ single-qubit gates, each either an IDENTITY gate or a NOT gate. We call such stack a NOT stack. 
E.g. the product (4) represents the circuit cascade

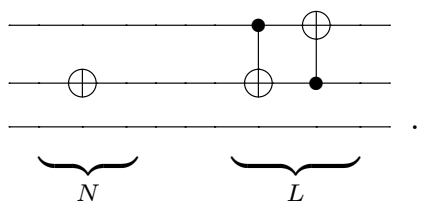

In general, $N$ consists of 0 to $w$ NOTs and $L$ consists of $\mathcal{O}\left(w^{2}\right)$ or $\mathcal{O}\left(\frac{w^{2}}{\log (w)}\right)$ controlled NOTs, depending on the synthesis method applied [13] [14].

\section{The Birkhoff decomposition of the two ZU matrices}

Because a member $Z$ of the group $\mathrm{ZU}(n)$ is diagonal, it cannot be decomposed as a weighted sum $\sum c_{j} P_{j}$ of permutation matrices $P_{j}$, such that the weight $\operatorname{sum} \sum c_{j}$ equals 1 . Indeed, if $\sum c_{j}=1$, then all line sums of the matrix $\sum c_{j} P_{j}$ are equal to 1 . Except for the $n \times n$ unit matrix, no diagonal matrix has this property. For this reason, we decompose the matrices $Z_{1}$ and $Z_{2}$ of (1) according to

$$
Z_{1}=G X_{1} G^{-1} \text { and } Z_{2}=G X_{2} G^{-1},
$$

where $G$ is a constant $n \times n$ (dephased) Hadamard matrix [16]. As the unitary matrices $Z_{1}$ and $Z_{2}$ have unit upper-left entry, automatically, $X_{1}$ and $X_{2}$ (equal to $G^{-1} Z_{1} G$ and $G^{-1} Z_{2} G$, respectively) have all line sums equal to 1 .

If $n=2^{w}$, we choose the following Hadamard matrix:

$$
G=H \otimes H \otimes \ldots \otimes H,
$$

i.e. the Kronecker product of $w$ small (i.e. $2 \times 2$ ) Hadamard matrices

$$
H=\frac{1}{\sqrt{2}}\left(\begin{array}{rr}
1 & 1 \\
1 & -1
\end{array}\right) \text {. }
$$

The matrix $G$ has following entries:

$$
G_{a, b}=\frac{1}{\sqrt{2^{w}}}(-1)^{f(a, b)},
$$

where $f(x, y)$ is the sum of the bitwise product of the binary numbers $x$ and $y$ and hence the matrix product of the row vector $x^{T}$ and the column vector $y$ :

$$
f(x, y)=\sum_{j} x_{j} y_{j} \bmod 2=x^{T} y .
$$

With this choice of $G$, the two matrix decompositions (5) represent the following circuit decomposition:

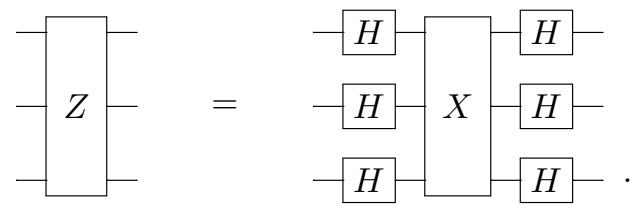


As the unitary matrices $Z_{1}$ and $Z_{2}$ are diagonal, automatically, $X_{1}$ and $X_{2}$ are epicirculant with unit pitch matrix. Indeed, if a $2^{w} \times 2^{w}$ matrix $D$ is diagonal and $G$ is given by (6-7), then an arbitrary entry of the product $G^{-1} D G$ is given by

$$
\begin{aligned}
\left(G^{-1} D G\right)_{j, k} & =\sum_{r} \sum_{s}\left(G^{-1}\right)_{j, r} D_{r, s} G_{s, k} \\
& =\sum_{r} \frac{1}{\sqrt{2^{w}}}(-1)^{-r^{T} j} D_{r, r} \frac{1}{\sqrt{2^{w}}}(-1)^{r^{T} k} \\
& =\frac{1}{2^{w}} \sum_{r}(-1)^{r^{T}(k-j)} D_{r, r} .
\end{aligned}
$$

We note that $\left(G^{-1} D G\right)_{0, k-j}=\frac{1}{2 w} \sum_{r}(-1)^{r^{T}(k-j)} D_{r, r}$ equals $\left(G^{-1} D G\right)_{j, k}$, which means that $G^{-1} D G$ is epicirculant according to Definition 1 with $x$ equal to the $w \times w$ unit matrix.

Any $2^{w} \times 2^{w}$ epicirculant matrix $M$ satisfies

$$
M=\sum_{m=0}^{2^{w}-1} M_{0, m} F_{m},
$$

with $F_{m}$ the epicirculant permutation matrix with shift vector equal to $m$ and same pitch matrix as $M$. Hence, $X_{1}$ and $X_{2}$ satisfy the (short) Birkhoff sums:

$$
X_{1}=\sum_{j=1}^{2^{w}} a_{j} E_{j} \text { and } X_{2}=\sum_{j=1}^{2^{w}} b_{j} E_{j},
$$

where the $E_{j}$ are the epicirculant permutation matrices with unit pitch matrix. Because $X_{1}$ and $X_{2}$ are unitary, we immediately have $\sum\left|a_{j}\right|^{2}=\sum\left|\left(X_{1}\right)_{0, j}\right|^{2}=1$ and $\sum\left|b_{j}\right|^{2}=\sum\left|\left(X_{2}\right)_{0, j}\right|^{2}=1$. Moreover, because both $X_{1}$ and $X_{2}$ have row sums equal to 1 , we have $\sum a_{j}=\sum\left(X_{1}\right)_{0, j}=1$ and $\sum b_{j}=\sum\left(X_{2}\right)_{0, j}=1$.

The unit-pitch epicirculant permutation matrices form a group isomorphic to the direct product $\left(\mathbf{C}_{2}\right)^{w}$ of order $2^{w}$. According to Lemma 3 , such permutation matrix $E_{j}$ represents a NOT stack.

\section{The Birkhoff decomposition of the scalar factor}

The unit-modulus scalar $a$ in (1) is to be interpreted as a $2^{w} \times 2^{w}$ unitary matrix $A$, i.e. $a$ times the $2^{w} \times 2^{w}$ unit matrix. It thus is also $a$ times the $2^{w} \times 2^{w}$ identity permutation matrix. Therefore it is a weighted 'sum' of permutation matrices:

$$
A=\sum_{j} d_{j} P_{j}=a P_{1} .
$$

We have $\sum\left|d_{j}\right|^{2}=|a|^{2}=1$; however, the sum $\sum_{j} d_{j}=a$ usually is not equal to 1 . 
The matrix $A$ equals the Kronecker product

$$
I \otimes I \otimes I \otimes \ldots \otimes I \otimes\left(\begin{array}{ll}
a & 0 \\
0 & a
\end{array}\right) \otimes I \otimes I \otimes I \otimes \ldots \otimes I,
$$

with $w-1$ appearances of the factor $I=\left(\begin{array}{ll}1 & 0 \\ 0 & 1\end{array}\right)$ and, within the product, arbitrary position of the factor $\left(\begin{array}{ll}a & 0 \\ 0 & a\end{array}\right)$. The scalar $a$ thus represents a $w$-qubit quantum circuit with merely one single-qubit gate acting on an arbitrary wire.

\section{The Birkhoff decomposition of the U matrix}

From the above discussion, we see that any $w$-qubit quantum circuit can be constructed as the following cascade:

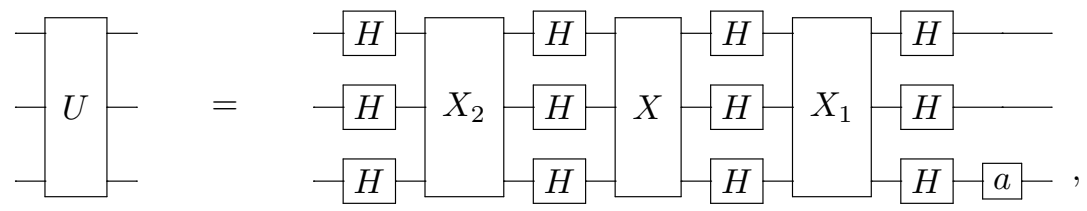

containing

$-4 w+1$ single-qubit gates represented by $\mathrm{U}(2)$ matrices:

- $4 w$ HADAMARD gates and

- one PHASE-SHIFT gate

and

- three $w$-qubit circuits represented by $\mathrm{XU}\left(2^{w}\right)$ matrices:

- one decomposable as a weighted sum of classical reversible circuits consisting of 2-bit gates (controlled NOT gates) and single-bit gates (NOT gates) and

- two decomposable as a weighted sum of classical reversible circuits consisting exclusively of single-bit gates (NOT gates).

We have

$$
\begin{aligned}
U & =a Z_{1} X Z_{2} \\
& =a G X_{1} G^{-1} X G X_{2} G^{-1} \\
& =a G\left(\sum_{j_{1}=1}^{2^{w}} a_{j_{1}} E_{j_{1}}\right) G^{-1}\left(\sum_{j=1}^{N(w)} c_{j} E_{j}\right) G\left(\sum_{j_{2}=1}^{2^{w}} b_{j_{2}} E_{j_{2}}\right) G^{-1} \\
& =a \sum_{j_{1}=1}^{2^{w}} \sum_{j=1}^{N(w)} \sum_{j_{2}=1}^{2^{w}} a_{j_{1}} c_{j} b_{j_{2}} G E_{j_{1}} G^{-1} E_{j} G E_{j_{2}} G^{-1}
\end{aligned}
$$

We note that the identities

$$
H \quad=
$$


and

$$
-H \oplus H-\quad=\quad-Z
$$

imply that each of the two compositions $G E_{j_{1}} G^{-1}$ and $G E_{j_{2}} G^{-1}$ can be replaced by a stack of $w$ gates, each either an IDENTITY gate, representing the unit matrix $\left(\begin{array}{ll}1 & 0 \\ 0 & 1\end{array}\right)$, or a $\mathrm{Z}$ gate, representing the matrix

$$
Z=\left(\begin{array}{rr}
1 & 0 \\
0 & -1
\end{array}\right)
$$

Thus:

Lemma 4 A NOT stack sandwiched between two HADAMARD stacks is a Z stack.

The $\mathbf{Z}$ stacks form a group isomorphic to $\left(\mathbf{C}_{2}\right)^{w}$ and are represented by diagonal $2^{w} \times 2^{w}$ matrices with an upper-left entry equal to 1 and all other diagonal entries equal to \pm 1 . Thus the matrix $G E_{j_{1}} G^{-1} E_{j} G E_{j_{2}} G^{-1}$ within eqn (8) represents an epicirculant permutation matrix sandwiched between two $\mathrm{Z}$ stacks and hence is a signed epicirculant permutation matrix. We summarise the present section by a new theorem:

Theorem 5 Every $U\left(2^{w}\right)$ matrix $U$ can be decomposed as

$$
U=a \sum_{j=1}^{M(w)} c_{j} S_{j},
$$

where $a$ is a complex (unit-modulus) scalar, where $j$ runs over $2^{w} \times 2^{w}$ signed epicirculant permutation matrices $S_{j}$, where $c_{j}$ are complex numbers, such that both $\sum c_{j}=1$ and $\sum\left|c_{j}\right|^{2}=1$, and $M(w)$ equals $4^{w} \times 2^{w}\left(2^{w}-1\right)\left(2^{w}-2\right)\left(2^{w}-\right.$ $\left.2^{2}\right) \ldots\left(2^{w}-2^{w-1}\right)$.

\section{Conclusion}

We conclude that an arbitrary quantum computer can be regarded as a weighted sum of almost-classical reversible computers. Each of these reversible computers consists of two surprisingly simple classical parts:

- one linear circuit (composed of exclusively controlled NOTs) and

- one NOT stack

and three small quantum parts:

- one complex scalar and

- two Z stacks.

Whereas a matrix product represents a circuit cascade, a matrix sum does not represent a simple circuit structure. Recently, there have been some attempts [17] [18] to apply a weighted matrix sum for quantum circuit synthesis. However, this so-called 'reuse method' is only efficient (in terms of gate count and ancilla count) in very specific cases. Further research may reveal the full impact of the unitary Birkhoff theorems on quantum computation. Future work may lead to applications in simulation of quantum systems by means of classical computers. 


\section{Acknowledgement}

Support by the European Cost Action IC 1405 'Reversible computation' is greatly acknowledged.

\section{References}

1. G. Birkhoff, "Tres observaciones sobre el algebra lineal", Universidad Nacional de Tucumán: Revista Matemáticas y Física Teórica, vol. 5 (1946), pp. 147-151.

2. M. Nielsen and I. Chuang, Quantum computation and quantum information, ISBN 9780521635035, Cambridge University Press, Cambridge (2000).

3. A. De Vos, Reversible computing, ISBN 9783642295164, Wiley - VCH, Weinheim (2010).

4. A. De Vos and S. De Baerdemacker, "The NEGATOR as a basic building block for quantum circuits", Open Systems 8 Information Dynamics, vol. 20 (2013), 1350004.

5. A. De Vos and S. De Baerdemacker, "On two subgroups of U $(n)$, useful for quantum computing", Journal of Physics: Conference Series: Proceedings of the 30 th International Colloquium on Group-theoretical Methods in Physics, Gent (July 2014), vol. 597 (2015), 012030.

6. M. Idel and M. Wolf, "Sinkhorn normal form for unitary matrices", Linear Algebra and its Applications, vol. 471 (2015), pp. 76-84.

7. A. De Vos and S. De Baerdemacker, "Scaling a unitary matrix", Open Systems $\&$ Information Dynamics, vol. 21 (2014), 1450013.

8. A. De Vos, S. De Baerdemacker, and Y. Van Rentergem, Synthesis of quantum circuits versus synthesis of classical reversible circuits, ISBN 9781681733814, Morgan \& Claypool, La Porte (2018).

9. S. De Baerdemacker, A. De Vos, L. Chen, and L. Yu, "The Birkhoff theorem for unitary matrices of arbitrary dimension", Linear Algebra and its Applications, vol. 514 (2017), pp. 151-164.

10. A. De Vos and S. De Baerdemacker, "The Birkhoff theorem for unitary matrices of prime dimension", Linear Algebra and its Applications, vol. 493 (2016), pp. $455-468$.

11. A. De Vos and S. De Baerdemacker, "The Birkhoff theorem for unitary matrices of prime-power dimension", Linear Algebra and its Applications, vol. 578 (2019), pp. $27-52$.

12. wikipedia, "Affine group", https://wikipedia.org/wiki/Affine_group (2018).

13. T. Beth and M. Rötteler, "Quantum algorithms: applicable algebra and quantum physics", In: G. Alber, T. Beth, M. Horodecki, P. Horodecki, R. Horodecki, M. Rötteler, H. Weinfurter, R. Werner, and A. Zeilinger, Quantum information, ISBN 3540416668, Springer Verlag, Berlin (2001), pp. 96-150.

14. K. Patel, I. Markov, and J. Hayes, "Optimal synthesis of linear reversible circuits", Quantum Information and Computation, vol. 8 (2008), pp. 282-294.

15. A. De Vos and S. De Baerdemacker, "Decomposition of a linear reversible computer: digital versus analog", International Journal of Unconventional Computing, vol. 6 (2010), pp. 239-263.

16. W. Tadej and K. Życzkowski, "A concise guide to complex Hadamard matrices", Open Systems $\&$ Information Dynamics, vol. 13 (2006), pp. 133-177. 
17. A. Klappenecker and M. Rötteler, "Quantum software reusability", International Journal of Foundations of Computer Science, vol. 14 (2003), pp. 777-796.

18. C. Allouche, M. Baboulin, T. Goubault de Brugière, and B. Valiron, "Reuse method for quantum circuit synthesis", International Conference on Applied Mathematics, Modeling and Computational Science, Waterloo (August 2017). 